QUARTERLY OF APPLIED MATHEMATICS

VOLUME LXVIII, NUMBER 1

MARCH 2010, PAGES 5-16

S 0033-569X(09)01176-6

Article electronically published on October 20, 2009

\title{
THE MATHEMATICS OF W. A. STRAUSS AND HIS CONTRIBUTIONS TO ANALYSIS
}

\author{
BY
}

\author{
MANOUSSOS GRILLAKIS
}

Department of Mathematics, University of Maryland, College Park, Maryland

The purpose of this note is to describe some of Walter Strauss's mathematical contributions and their impact to the next generation of mathematicians. The choice of articles is my personal preference and it includes work that influenced me, as well as some of my friends and collegues. This presentation is by no means a complete description of Walter's work, which consists of more than one hundered published papers on a wide range of subjects. It is my hope that it contains a flavor of his work and furthermore gives an idea of Walter's originality. One can find further references and subsequent work in the books cited in the bibliography. Let us start by some general information about Walter. Here are the bare facts.

1) Walter Alexander Strauss, Ph.D. 1962. Advisor: Irving Segal.

2) Dissertation: Scattering for Hyperbolic Equations

3) Mathematical interests: Nonlinear Waves

4) Students: 20 Ph.D. students so far.

In order to add some more details, let me cite Walter's mathematical interests.

1) Partial Differential Equations

2) Mathematical Physics

3) Stability Theory

4) Solitary Waves

5) Kinetic Theory of Plasmas

6) Scattering Theory

7) Water Waves

8) Dispersive Waves

Before we leave these introductory remarks, it is perhaps worth mentioning a recent Science-Watch citation of April 4, 2004 on a paper of Walter Strauss written in collaboration with A. Constantin,

- SCIENCE-WATCH: Exact Steady Periodic Water Waves with Vorticity, Comm. Pure Appl. Math. 57, April 2004 (with A. Constantin)

Received December 31, 2008.

2000 Mathematics Subject Classification. Primary 35L05, 35L10, 35L60, 43A15, 43A85, 35Q55.

E-mail address: mng@math.umd.edu

(C)2009 Brown University 
(1) Linear Scattering: The first papers that I would like to mention are concerned with Walter's early work in Scattering Theory, in collaboration with J. Cooper,

Scattering of waves by periodically moving bodies, J. Funct. Anal. 47 (1982) (with J. Cooper)

In a sequence of papers, W. Strauss and J. Cooper considered the following types of problems.

a) The wave equation in the exterior of a moving obstacle,

$$
\square \phi=0 ; \quad x \in \Omega(t)
$$

with Dirichlet boundary conditions and periodic motion,

$$
\phi=0 ; \quad x \in \partial \Omega(t) ; \quad \Omega(t+T)=\Omega(t) .
$$

b) The wave equations with a time periodic potential,

$$
\square \phi+V(t, x) \phi=0 \quad \text { with } \quad V(t+T, x)=V(t, x) .
$$

The problem is to study the asymptotic behaviour of the solutions as $t \rightarrow \pm \infty$. A more precise question is to relate the asymptotic behaviour of the solutions to that of a standard problem, such as the solutions of the free wave equation without an obstacle. Walter Strauss and Jeff Cooper were motivated by applications but they were inspired by the work of Lax and Phillips who developed earlier a general framework of scattering theory for static objects. In a series of papers they developed the definitive scattering theory for moving obstacles. What is the scattering operator? Suppose that one can demonstrate that there exist two solutions of the free equations, say $\square \phi_{ \pm}=0$, such that $\lim _{t \rightarrow \pm \infty}\left\|\phi(t)-\phi_{ \pm}(t)\right\|=0$, respectively. Then one can think of the scattering map as $S: \phi_{-} \mapsto \phi_{+}$. Care is needed in this to check that $\phi_{-}$is an incoming solution and $\phi_{+}$is outgoing, and of course the issue is to check that $S$ is a well-defined map between appropriate function spaces.

Walter and Jeff established the following fundamental description. In the absence of trapped rays, there is a sequence of complex numbers called scattering frequencies with imaginary parts tending to $+\infty$ and real parts in the interval $[0,2 \pi / T]$. Each scattering frequency has attached a nontrivial outgoing solution of the form $\exp \left[i \sigma_{j} t\right] p_{j}(x, t)$ with $p_{j}(x, t)$ periodic (or pseudoperiodic) in time. The following asymptotic expansion holds:

$$
\phi(x, t) \sim \sum_{j} c_{j} e^{i \sigma_{j} t} p_{j}(x, t) \quad \text { locally in space. }
$$

Moreover, concerning the scattering amplitude, they gave the following description. Let $(\sigma, \omega)$ be the incident frequency and direction of a plane wave and $\left(\sigma^{\prime}, \omega^{\prime}\right)$ the reflected (distorted) plane wave. There exist functions, $A_{m}$, meromorphic in the complex plane, with poles at scattering frequencies, $\sigma_{j}+2 \pi n / T$, and the following expansion holds:

$$
K\left(\sigma^{\prime}, \omega^{\prime} ; \sigma, \omega\right)=\sum_{m} A_{m}\left(\sigma, \omega^{\prime}, \omega\right) \delta\left(\sigma^{\prime}-\sigma-2 \pi m / T\right) .
$$

(2) Nonlinear Scattering: The next paper of Walter that I would like to discuss is one which he wrote in collaboration with Cathleen Morawetz, and is concerned with the Nonlinear (cubic) Klein-Gordon equation. 
- Decay and scattering of solutions of a nonlinear relativistic wave equation (with Cathleen Morawetz), Comm. Pure Appl. Math. 25, 26 (1972-73)

The nonlinear (cubic) Klein-Gordon equation is

$$
\square \phi+m \phi+a \phi^{3}=0 \text {. }
$$

Walter and Cathleen studied the asymptotic behaviour of this equation; namely, they established the existence of a scattering operator in the spirit described previously. Here is an outline of the method.

All known methods that study the large time behaviour of nonlinear dispersive equations start with Morawetz's radial estimate. It can be described as follows. The energymomentum tensor which satisfies the system

$$
\nabla_{\mu} T_{\nu}^{\mu}=0
$$

is combined with a structure equation. If we define $\rho:=\phi^{2} / 2$, then this density $\rho$ satisfies

$$
\square \rho+\lambda+a \phi^{4} / 2=0,
$$

where $\lambda$ is the Lagrangian density. Contraction of the energy-momentum equation with a vector field $X^{\nu}$ yields

$$
\nabla_{\mu}\left\{T_{\nu}^{\mu} X^{\nu}\right\}=T_{\nu}^{\mu} \nabla_{\mu} X^{\nu}
$$

and an apropriate choice of a vector field, combined with the structure equation, gives an a priori estimate that reads

$$
\sup _{\vec{x}_{0}} \int_{R^{3+1}} \frac{\phi^{4}(x)}{\left|\vec{x}-\vec{x}_{0}\right|} d x \leq C E,
$$

where $E$ is the conserved energy of the system. The particular estimate follows from contraction of the energy-momentum tensor with the vector field

$$
X:=\left(1, \frac{\vec{x}}{|\vec{x}|}\right),
$$

which is characteristic.

This paper is a tour de force. Here are the basic steps in the analysis of Walter and Cathleen. First translate the estimate to something like

$$
\int_{B_{t \log t} \times I} \phi^{4} d x \leq \epsilon,
$$

where $|I|=T$, for some fixed large length interval, and $\epsilon$ small. It should be understood that $\epsilon$ plays the role of a threshold, $T$ is some fixed large length and the integral is taken in $I \subset\left[T_{0},+\infty\right)$. Now the following sequence of steps is established, improving on the original estimate.

1) Smallness most of the time,

$$
\sup _{\vec{x}}|\phi(t, \vec{x})| \text { is small in arbitrarily large time intervals. }
$$

2) Decay in time,

$$
\phi(t, \vec{x}) \rightarrow 0 \quad \text { uniformly as } \quad t \rightarrow+\infty \text {. }
$$


3) Integrability of the supremum norm in time,

$$
\int d t\left\{\sup _{\vec{x}} \phi^{2}(t, \vec{x})\right\}
$$

4) Decay in time of the supremum norm,

$$
\sup _{\vec{x}}|\phi(t, \vec{x})| \leq \frac{C}{t^{3 / 2}} .
$$

From the last decay estimate one can prove scattering. This paper served as an inspiration to many, including me. Let me mention here another related paper, which is also fundamental in my opinion.

- Decay and scattering of solutions of the nonlinear Schrödinger equation (with Jeng-Eng Lin), J. Funct. Anal. 30 (1978)

The authors of the paper above consider the defocusing nonlinear Schrödinger equation,

$$
i \partial_{t} \psi-\Delta \psi+|\psi|^{2 p-2} \psi=0
$$

and establish a Morawetz type estimate,

$$
\int_{R^{3+1}} \frac{|\psi(t, \vec{x})|^{p}}{\left|\vec{x}-\vec{x}_{0}\right|} d \vec{x} d t \leq \sqrt{E M},
$$

where $E$ is the energy and $M$ is the mass. This estimate is truly different than the one before. The energy-momentum tensor is not symmetric; however, we do have conservation of mass and a structure equation which reads

$$
-\Delta \rho+\lambda+c \rho^{p / 2}=0,
$$

where again $\rho$ is the density, $\lambda$ is the Lagrangian density and $c>0$. This estimate is named after C. Morawetz in that paper, but it should be called the Morawetz-Strauss-Lin estimate.

The papers mentioned above serve as the starting point for almost all scattering theorems, the pseudoconformal identity of Ginibre and Velo being the other essential ingredient. The same estimate, essentially, was also used to prove global existence for the energy critical Schrödinger equation by J. Bourgain in the radial case, and by Colliander, Keel, Stafillani, Tao and Takaoka without the radial assumption. There are reincarnations and repeated use of these methods in the works of J. Colliander, T. Tao, M. Visan, Killip, Zhang, Nakanishi, Ozawa, among others.

At this point it is worth saying a few words concerning Nonlinear Scattering. The field is vast. The original idea of this type of problem goes back to I. Segal. The first serious treatment of the subject is due to Walter Strauss and his collaborators. There are of course many other people involved. Let me mention a few: J. Ginibre and G. Velo and Y. Tsutsumi, T. Cazenave and also P. Brenner, H. Pecher, Von-Wahl, K. Yajima. After J. Bourgain's revolution, the field attracted the attention of T. Tao and his collaborators, and subsequent students. Y. Tsutsumi is responsible for creating a large Japanese group. Some more names with important contributions are F. Merle, C. Kenig, G. Ponce, and L. Vega. At this point I should mention F. John, who had some of the most original 
ideas in relation to the wave equation. Finally, an important body of work was created by B. Glassey and T. Sideris.

In order to appreciate how basic Walter's work was, let me mention the fact that if the nonlinearity is of the form $f(\psi):=a|\psi|^{p-1} \psi$, then the behaviour of the solution is governed by the four powers,

$$
1+\frac{2}{n}<\gamma(n)<1+\frac{4}{n}<1+\frac{4}{n-2},
$$

where $\gamma(n)$ ( $n$ is the dimension) is given by

$$
\gamma(n)=\frac{1}{2}+\frac{1}{n}+\left(\left(\frac{1}{2}+\frac{2}{n}\right)^{2}+\frac{2}{n}\right)^{\frac{1}{2}}
$$

and is now called the Strauss number.

(3) Conformal Wave Equation: This is an early one of Walter's papers, which appeared in 1968, and again it opened a whole line of similar investigations.

- Decay and asymptotics for $\square u=F(u)$, J. Funct. Anal. 2 (1968)

In this paper, Walter considers the conformally invariant wave equation in $3+1$ dimensions,

$$
\square \phi+a \phi^{3}=0,
$$

which is invariant under dilations-scaling and inversions, namely the transformations

$$
x^{\mu} \mapsto \lambda x^{\mu} ; \quad x^{\mu} \mapsto \frac{x^{\mu}}{g_{\mu \nu} x^{\mu} x^{\nu}} ; \quad \phi \mapsto c \phi .
$$

The conformal energy is essentially the sum of squares of derivatives of the form

$$
D \phi:=x^{\mu} \nabla_{\mu} \phi ; \quad L_{\mu \nu} \phi:=\epsilon_{\mu \nu \lambda \rho} x^{\lambda} \nabla^{\rho} \phi,
$$

where $L_{\mu \nu}$ are Lorentz derivatives, and $\epsilon_{\mu \nu \lambda \rho}$ is the totally antisymmetric tensor. The essential estimate derived by Walter was the following:

$$
\int_{R^{3}} \phi^{4} d \vec{x}=O\left(t^{-2}\right) \text {. }
$$

In his paper mentioned above, Walter Strauss proved scattering in the conformal energy space. A different but equivalent approach is to use the Conformal Mapping method of R. Penrose, which maps the space-time to the Penrose diamond. The idea was suggested and used by D. Christodoulou in the 1980s and was also employed by J. Baez, I. Segal, and Z. Zhou. The same idea was further elaborated upon by J. Ginibre and G. Velo, von Wahl and H. Pecher, but I should emphasize that Walter's paper appeared in 1968.

(4) Linear Decay Estimates: Here is another fundamental paper of Walter.

- $L^{p}-L^{q}$ estimates for the Klein-Gordon equation, J. Math. Pures Appl. 59 (1980) (with B. Marshall and S. Wainger).

The authors consider the linear Wave or Klein-Gordon equations,

$$
\square \phi=0 \text { or } \square \phi+m \phi=0 \text {, }
$$

with data (for simplicity) $\phi(0, \vec{x})=0$ and $\phi_{, t}(0, \vec{x})=\phi_{1}$. They establish the following linear decay estimates: 
a) Wave Equation,

$$
\left\|R(t) \phi_{1}\right\|_{L^{q}} \leq \frac{C}{t^{a}}\left\|\phi_{1}\right\|_{L^{p}} ; \quad a=(n / p)+(n / q)-1,
$$

where $R(t)$ is the Riemann-Green propagator. For simplicity take $n=3$. Then the coordinates $(1 / p, 1 / q)$ form the triangle with vertices,

$$
(3 / 4,1 / 4) ; \quad(0,0) ; \quad(1,1) .
$$

b) For the Klein-Gordon equation, set $a=\min \{-2 / q,-3 / 2+3 / q\}$ for the most interesting case when $1 / p+1 / q=1$ and one obtains again a triangle.

This paper came soon after the ground-breaking work of Strichartz. The idea was to use complex interpolation, developed by E. Stein, which for the wave equation amounts to studying the kernel,

$$
R_{z}(t, \vec{x})=\frac{1}{\left(t^{2}-|\vec{x}|^{2}\right)_{+}^{z}} ; \quad z \in \mathbf{C},
$$

where for example when $z=1$ the kernel is the delta function on the characteristic surface $t= \pm|\vec{x}|$. The Klein-Gordon case is considerably harder but follows the same path.

From these decay estimates one can obtain all the generalized Strichartz-type estimates with mixed norms. The only essential improvement since then is the end-point estimate of Keel-Tao. To see the rest (duality and the $T T^{*}$ argument), duality first,

$$
\left\langle g(t) \mid R(t) \phi_{1}\right\rangle=\left\langle R^{*}(t) g \mid \phi_{1}\right\rangle \text {, }
$$

and the $T T^{*}$ second,

$$
\left|\left\langle R^{*}(t) g(t) \mid \phi_{1}\right\rangle\right| \leq\left\|\phi_{1}\right\|_{2}\left\langle R(t) R^{*}(s) g(s) \mid g(t)\right\rangle^{1 / 2} .
$$

The rest of the argument consists of the use of $L^{p}-L^{q}$ estimates from the previous page and the Hardy-Littlewood-Polya inequality. With forcing, the estimates follow the same line of argument. The $T T^{*}$ argument is due to J. Ginibre and G. Velo, and I should mention that the Christ-Kiselev lemma can be used to simplify the method of proof.

These estimates had a tremendous career. At the time they were thought of as a novelty, but it turns out to be the most flexible of tools. The prehistory was the original paper of Strichartz for the diagonal $p=q$ and the Stein-Thomas restriction theorem for the Fourier Transform. These estimates were recast and used by myself, L. Kapitanski, P. Brenner, T. Tao, M. Keel, J. Shatah, M. Struwe, J. Ginibre and G. Velo, T. Cazenave, among many others. Today they form the cornerstone of the theory of Wave Equations. (5) Stability Theory: Walter's work that has so far the most citations is the following two papers.

- Stability theory of solitary waves in the presence of symmetry I,II, J. Funct. Anal. $\mathbf{7 4}$ (1987-90) (with J. Shatah and M. Grillakis).

The theory is based on two basic examples, namely the Klein-Gordon and Schrödinger equations

$$
\begin{aligned}
\square \phi+m^{2} \phi-a|\phi|^{2} \phi & =0 ; \quad \phi \in \mathbf{C}, \\
i \partial_{t} \psi-\Delta \psi-a|\psi|^{2} \psi & =0 ; \quad \psi \in \mathbf{C} .
\end{aligned}
$$


One is interested in stationary solutions of the form $\phi(t, \vec{x})=e^{i \omega t} \phi(\vec{x})$ or $\psi(t, \vec{x})=$ $e^{-i \omega t} \phi(\vec{x})$, and the resulting elliptic equations are

$$
\begin{gathered}
-\Delta \phi+\left(m^{2}-\omega^{2}\right) \phi-a \phi^{3}=0, \\
-\Delta \phi+\omega \phi-a \phi^{3}=0 .
\end{gathered}
$$

Both examples have a very rich history. T. D. Lee and his collaborators were considering systems of coupled wave equations that possess this type of solutions and which can be quantized and interpreted as elementary particles. The Schrödinger example comes up in optics, laser beams or optical fibers where, again, one considers systems of coupled equations; similar equations appear in Bose Einstein Condensation. The issue is whether these solutions are stable in an appropriate sense. Notice that one should consider orbital stability for the rotating solution.

Early work on this problem was done by J. Shatah when he was one of Walter's students. This was also part of my thesis. A sharp theory should examine criteria for instability as well. Stability results existed in the literature, for example in the work of T. D. Lee with Friedberg and Sirlin, as well as the original work of M. Weinstein. Walter started with an abstract formulation of this type of problems. Consider a Hilbert space $X$ and its dual $X^{*}$ with $E(u): X \mapsto R$ a functional and $J: X^{*} \mapsto X$ a skew adjoint operator. Thus $E$ is the Hamiltonian, and the evolution equation is described by

$$
\frac{d u}{d t}=J E^{\prime}(u) ; \quad u \in X
$$

Now the important point is that we have a Lie group which acts unitarily on $X$ and preserves the energy $E$. If $T(g)$ is the action, then $E(T(g) u)=E(u)$. This generates conserved quantities called "charge",

$$
Q_{\omega}(u):=\left\langle J^{-1} T_{\omega} u \mid u\right\rangle ; \quad \omega \in \mathcal{G},
$$

where the pairing is between the dual spaces, $\left\langle X^{*} \mid X\right\rangle$. In this framework, solitary waves are solutions of the form $u(t)=T\left(e^{t \omega}\right) \phi_{\omega}$, where $e^{t \omega}$ is the orbit generated by some $\omega \in \mathcal{G}$ in the Lie Algebra of $G$. They satisfy the abstract equation,

$$
E^{\prime}\left(\phi_{\omega}\right)=J^{-1} T_{\omega} \phi_{\omega} ; \quad J^{-1}: X \mapsto X^{*} .
$$

Orbital stability is concerned with the stability of the orbit,

$$
\left\{u \mid Q_{\rho}(u)=Q_{\omega}\left(\phi_{\omega}\right) ; \rho \in \mathcal{G}_{\omega}\right\},
$$

where $\mathcal{G}_{\omega}$ is the centralizer of $\omega$. Linearizing, one obtains the equation

$$
\frac{d v}{d t}=J \mathbf{H} v ; \quad \mathbf{H}:=E^{\prime \prime}\left(\phi_{\omega}\right)-Q_{\omega}^{\prime \prime}\left(\phi_{\omega}\right) .
$$

Now form the quantity

$$
d(\rho):=E\left(\phi_{\rho}\right)-Q_{\rho}\left(\phi_{\rho}\right) ; \quad \rho \in \mathcal{G}_{\omega},
$$

and the criterion is as follows:

$$
\begin{aligned}
& \text { if neg. eigenv. }(\mathbf{H})-\text { pos. eigenv. }\left(d^{\prime \prime}\right)=0, \quad \phi_{\omega} \quad \text { is stable, } \\
& \text { if neg. eigenv. }(\mathbf{H})-\text { pos. eigenv }\left(d^{\prime \prime}\right)=\text { odd } \quad \phi_{\omega} \quad \text { is unstable; }
\end{aligned}
$$


otherwise stability is undecided.

This characterization is actually optimal in this generality. Because this framework is so general it applies to many examples, optical fibers being one; hence it generated attention and has a very large number of citations. At this point I should mention the work of C. Jones which was complementary as well as the work of M. Weinstein. The main difficulty is analyzing the spectral properties of $\mathbf{H}$. One can extend this (but it is nontrivial) to water wave type equations, KDV and Boussinesq, beam equations, etc.; see for example the work of Strauss-Souganidis, S. Levandosky, Yue Liu, P. Karageorgis among many others as well as C. Jones, J. Bona, Moloney, T. Kapitula, and Pelinovski. (6) Plasma: Here are two beautiful papers of Walter written in collaboration with R. Glassey.

- Singularity formation in a collisionless plasma could occur only at high velocities, Arch. Rational Mech. Anal. 92 (1986) (with R. Glassey)

- Absence of shocks in an initially dilute collisionless plasma, Comm. in Math. Phys. 113 (1987) (with R. Glassey)

Walter and Bob consider collisionless plasma of charged particles with relativistic velocities; i.e., the relativistic velocity is given by $\vec{v}_{r}:=c \vec{v} / \sqrt{c^{2} m^{2}+|\vec{v}|^{2}}$, and for charged particles with charge $q$, the motion is described by the equations

$$
\frac{d \vec{x}}{d t}=\vec{v}_{r} ; \quad \frac{d \vec{v}}{d t}=q\left(\vec{E}+c^{-1} \vec{v}_{r} \wedge \vec{B}\right) .
$$

Now introduce the microlocal density $f(t, \vec{x}, \vec{v})$ which satisfies the Vlasov-Maxwell equation,

$$
\partial_{t} f+\vec{v}_{r} \cdot \vec{\nabla}_{x} f+q\left(\vec{E}+c^{-1} \vec{v}_{r} \wedge \vec{B}\right) \cdot \vec{\nabla}_{v} f=0 .
$$

Charge density $\rho$ and current $\vec{j}$ are the velocity averages,

$$
\rho=(4 \pi) \int d \vec{v}\{q f\} ; \quad \vec{j}=(4 \pi) \int d \vec{v}\left\{q \vec{v}_{r} f\right\},
$$

and the electromagnetic field is generated by Maxwell's equation,

$$
\begin{aligned}
\partial_{t} \vec{E} & =c \vec{\nabla} \wedge \vec{B}-\vec{j} ; \quad \vec{\nabla} \cdot \vec{E}=\rho, \\
\partial_{t} \vec{B} & =-c \vec{\nabla} \wedge \vec{E} ; \quad \vec{\nabla} \cdot \vec{B}=0 .
\end{aligned}
$$

One wishes to solve this system after prescribing some intial data, say $f_{0}, \vec{E}_{0}$ and $\vec{B}_{0}$. The basic question is whether a solution exists for all time.

Here is an outline of the results in those two papers.

- Control of high velocities, i.e. a bound of the form

$$
\sup _{0 \leq t \leq T} \sup _{\vec{x}} \int d \vec{v}\{|\vec{v}| f\} \leq C(T)
$$

implies global existence and regularity.

- Almost neutral plasma, i.e. the smallness assumption

$$
\left\|\vec{E}_{0}\right\|_{C^{2}}+\left\|\vec{B}_{0}\right\|_{C^{2}}+\left\|\sum_{a} q_{a} m_{a}^{3} f_{a, 0}\right\|_{C^{1}}<\epsilon,
$$

implies global existence and regularity, for several species of particles. 
These papers are another tour de force. The idea is to write the wave equation for $(\vec{E}, \vec{B})$, for example,

$$
\square \vec{E}=-\vec{\nabla} \rho-\partial_{t} \vec{j},
$$

and use the Riemann-Green propagator, which can be expressed as an integral over the backwards characteristic cone,

$$
\vec{E}(t, \vec{x})=\vec{E}_{L}(t, \vec{x})+\int_{K(t, \vec{x})} d S \int d \vec{v}\{D f\},
$$

where $D f$ denotes derivatives of the density. Now the vector fields,

$$
S:=\partial_{t}+v_{r}^{k} \partial_{k} ; \quad T_{j}:=-\omega_{j} \partial_{t}+\partial_{j} ; \omega_{j} \text { the angular variable, }
$$

which are linearly independent if $\left|\vec{v}_{r}\right|<1-\delta$, can be used to expand $D f$. The $T_{j}$ are tangential to the cone $K$ and can be integrated by parts. For $S$-type derivatives the authors use Vlasov's equation

$$
S f=-q \vec{\nabla}_{v}\left[\left(\vec{E}+c^{-1} \vec{v}_{r} \wedge \vec{B}\right) f\right] .
$$

The strategy is to obtain classical $L^{\infty}$ bounds of the solution in order to use them in a bootstrap construction.

R. DiPerna and P.L. Lions proved global existence of weak solutions (without uniqueness). There is substantial progress for the Vlasov-Poisson system in the work of P.L. Lions and B. Perthame. R. Glassey and J. Schaffer developed the ideas in these papers in a series of papers. Later, Yan Guo with W. Strauss, and also Zhiwu Lin with W. Strauss, gave the first rigorous study of instability for special solutions. Kinetic equations became very popular in the last twenty years, because they can be considered as an intermediate model between a microscopic and a macroscopic description of fluids. Despite all the progress, the results of Walter Strauss and Bob Glassey are the best in the literature within the framework of classical solutions.

(7) Instability in Plasma Equilibria: Another paper of Walter which is a first of its type is the following.

- Instability of periodic BGK equilibria, Comm. Pure Appl. Math. 48 (1995) (with Yan Guo).

The problem considered by Walter and Yan is the one-dimensional version of a relativistic Maxwell-Vlasov system (two charged particles \pm ):

$$
\begin{aligned}
\partial_{t} f_{ \pm} & +v_{r} \partial_{x} f_{ \pm} \pm E \partial_{v} f_{ \pm}=0, \\
\partial_{t} E & =-j ; \quad \partial_{x} E=\rho .
\end{aligned}
$$

This system has many nontrivial solutions found by Bernstein, Greene and Kruskal (BGK):

$$
\begin{aligned}
f_{ \pm} & =\mu_{ \pm}(\langle u\rangle \mp \Phi(x)), \\
E & =\Phi^{\prime}(x) .
\end{aligned}
$$

This paper offers the first rigorous proof that the BGK solutions are nonlinearly unstable provided that $\mu_{+}+\mu_{-}$is sufficiently nonmonotone. There is preliminary work by O. Penrose on this problem. The idea was to understand first the linearized equation, i.e. 
to prove that there is a growing mode, and then apply a careful analysis of the nonlinear equation.

A related result which is again notable for its originality is the following article.

- Stability of semiconductor states with insulating and contact boundary conditions, Arch.

Rational Mech. Anal. 179 (2005) (with Y. Guo)

The authors look at a hydrodynamic model for semiconductors,

$$
\begin{aligned}
\rho_{t}+\nabla \cdot(\rho u) & =0, \\
(\rho u)_{t}+\nabla \cdot(\rho u \otimes u)+\nabla[P(\rho)]-\rho E & =-\rho u / \tau, \\
\nabla \cdot E & =\rho-\bar{\rho} .
\end{aligned}
$$

Here $\rho$ is the density of charged carriers, $P$ is the pressure, $E$ is the electric field and $u$ is the velocity. The parameter $\tau$ is a time relaxation parameter (assumed small). $\bar{\rho}(x)$ is the background static density profile. Now introduce the electrostatic potential $\nabla \Phi=E$, and a realistic model must be considered in some bounded domain with boundary conditions as follows. Either specify $\rho$ or $\Phi$ on the contact parts or

$$
u \cdot \nu \text { and } \quad \nabla \Phi \cdot \nu=0
$$

on the insulating parts, $\nu$ being the unit normal vector on the boundary. Now consider steady states $\left(\rho_{0}, u_{0}, \Phi_{0}\right)$ which are known to exist (Degond, Markowich) and the question is whether these solutions are stable in an appropriate sense.

W. Strauss and Y. Guo prove global existence of solutions that are small perturbations of the steady state. The background, $\rho_{0}, \Phi_{0}$ and $\bar{\rho}$ are not assumed to be small but $u_{0}=0$. Thus they establish stability of these solutions. This is the first result that treats the three-dimensional problem with boundary conditions. Before this, there were results in one space dimension or in three without boundary. The idea was to write the equations as a symmetric hyperbolic system in such a way so that it is compatible with the boundary conditions and it is amenable to analysis.

(8) Water Waves: Here is the paper cited in Science-Watch.

- Exact steady periodic water waves with vorticity, Comm. Pure Appl. Math. LVII (2004) (with A. Constantin)

Walter and Adrian consider an incompressible fluid, $u_{x}+v_{y}=0$, and Euler's equations

$$
\begin{aligned}
& u_{t}+u u_{x}+v u_{y}=-P_{x}, \\
& v_{t}+u v_{x}+v v_{y}=-P_{y}-g .
\end{aligned}
$$

Now $y=\eta(t, x)$ is the free surface profile and the boundary conditions are

$$
P(x, \eta(t, x))=P_{a t m} ; \quad v(t, x, \eta(t, x))=n_{t}+u(t, x \eta(t, x)) \eta_{x} .
$$

At the bottom of the channel, one has $v(t, x,-d)=0$. Suppose we want to find periodic travelling waves for the surface profile $\eta$ moving with speed $c$. The stream function,

$$
\psi_{x}=-v ; \quad \psi_{y}=u-c
$$


gives the vorticity equation $\Delta \psi=\omega$ and if $u<c$, there exists a $\gamma(\psi)$ vorticity function such that $-\Delta \psi=\gamma(\psi)$. The relative mass flux is expressed by

$$
p_{0}:=\int_{-d}^{\eta(x)}[u(x, y)-c] d y \text { and } \Gamma(p)=\int_{0}^{p} \gamma(-s) d s .
$$

The authors establish the following basic result.

- For speed $c>0$ and wavelength $L>0$ with $p_{0}<0$, if the vorticity function $\gamma \in C^{1+\alpha}$ satisfies the condition

$$
\int_{p_{0}}^{0}\left[\frac{4 \pi^{2}\left(p-p_{0}\right)^{2}}{L^{2}}\left(2 \Gamma(p)-2 \Gamma_{\min }\right)^{1 / 2}+\left(2 \Gamma(p)-2 \Gamma_{\min }\right)^{3 / 2}\right] d p<g p_{0}^{2},
$$

there exists a connected set $\mathcal{C}$ of solutions $(u, v, \eta)$ which contains the trivial laminar flows $\eta=0$ and there is a sequence $\left(u_{n}, v_{n}, \eta_{n}\right) \in \mathcal{C}$ for which $\max _{\bar{D}_{\eta_{n}}} u \rightarrow c$ (stagnation point).

This is a very old problem. For irrotational flows, the investigation of water waves was initiated by Stokes (1847). Rigorous constructions were offered by Nekrasov, LeviCivita and Struik (1920) via power series. Large solutions (again irrotational) were constructed by Krasovski and Keady-Norbury (1961-1978), respectively. For flows with vorticity there was almost nothing; Gerstner (1802) constructed an explicit solution in an infinite depth channel. Dubrail-Jacotin (1934) used power series to construct small waves. The construction of W. Strauss and A. Constantin is the first proof of large travelling water waves with vorticity.

The idea was to recast the problem using the stream function $\psi$ as one of the coordinates $\left(\psi_{y}>0\right)$ (this originated in the work of Dubrail-Jacotin), and write a quasilinear elliptic equation for the height function in these coordinates with a nonlinear boundary condition. The point is that the equations are now in a square. Subsequently they used the general bifurcation theory developed by P. Rabinowitz, actually a more recent version by Healey and Simpson which is suitable for nonlinear elasticity.

(9) Existence of Solitary Waves: This is another paper remarkable for its originality.

- Existence of solitary waves in higher dimensions, Comm. in Math. Phys. 55 (1977)

This is the first construction of solitary waves in higher dimensions. One wishes to solve the equation

$$
-\Delta \phi+m \phi-a \phi^{3}=0 ; \quad \vec{x} \in \mathbf{R}^{n} .
$$

An idea is to try to minimize the energy,

$$
E[\phi]:=\int_{R^{n}} d \vec{x}\left\{|\nabla \phi|^{2}+m^{2} \phi^{2}-\frac{a}{2} \phi^{4}\right\} .
$$

The natural space for this is $H^{1}\left(\mathbf{R}^{n}\right)$ and the minimization starts with some minimizing sequence $\left\{\phi_{n}\right\}_{n=1}^{\infty} \subset H^{1}$. We know that $\left\|\phi_{n}\right\|_{H^{1}} \leq C<+\infty$; hence (up to a subsequence) it converges weakly to some $\phi \in H^{1}$. The crucial issue is to check that $\phi \neq 0$ and

Walter assumed radial symmetry and proved that

$$
\int d \vec{x}\left\{\phi_{n}^{4}\right\} \rightarrow \int d \vec{x}\left\{\phi^{4}\right\}
$$

$$
H_{r}^{1} \subset_{\text {comp }} L^{p}, \quad \text { for } \quad p<p_{c},
$$


where $p_{c}$ is the critical Sobolev power.

This result generated a theory that examines how one might lose compactness if one removes the radial symmetry assumption; see for example the work P.L. Lions and H. Brezis as well as C. Taubes. For ground states (minimum energy), Steiner symmetrization can be combined with the result above. Ground states are necessarily positive and the work of Gidas-Ni-Nirenberg implies that they are actually radially symmetric (for a scalar equation). For $p=p_{c}$ the embedding is not compact, so the result is sharp.

(10) Existence of Nonlinear Waves: The last paper that I would like to mention is the following.

- On weak solutions of semi-linear hyperbolic equations, Anais Acad. Brasil. Cienc. 42 (1970)

Walter considers a general semilinear wave equation,

$$
\square \phi+f(\phi)=0 .
$$

The point is that the nonlinear term $f$ can be anything. Walter has a general and simple argument that constructs weak solutions for any type of nonlinearity. This is the only result for supercritical equations known so far. This method has been used by many other mathematicians; in particular, it was employed by J. Shatah for the construction of weak solutions for the wave map system.

\section{REFERENCES}

[1] J. Bourgain, Global solutions of nonlinear Schrödinger equations, American Mathematical Society, Providence RI, 1999. MR1691575 (2000h:35147)

[2] T. Cazenave, Semilinear Schrödinger equations, Courant Lecture Notes in Math. 10, AMS, 2003. MR:2002047 (2004j:35266)

[3] Walter A. Strauss, Nonlinear wave equations, CBMS Regional Conference Series in Mathematics, 73, AMS, 1989. MR1032250 (91g:35002)

[4] T. Tao, Nonlinear Dispersive Equations: Local and Global Analysis, CBMS Regional Conference Series in Mathematics, 106, AMS, 2006. MR.2233925 (2008i:35211) 